\title{
Combinatorial Geometries Representable over GF(3) and GF(q). I. The Number of Points*
}

\author{
Joseph P. S. Kung \\ Department of Mathematics, North Texas State University, Denton, TX 76203, USA
}

\begin{abstract}
Let $q$ be a prime power not divisible by 3 . We show that the number of points (or rank-1 flats) in a combinatorial geometry (or simple matroid) of rank $n$ representable over $\mathrm{GF}(3)$ and $\mathrm{GF}(q)$ is at most $n^{2}$. When $q$ is odd, this bound is sharp and is attained by the Dowling geometries over the cyclic group of order 2 .
\end{abstract}

\section{Introduction}

Our starting point is the classical theorem about the number of points in a regular combinatorial geometry due to Heller [7] (see also [1] and [12]). Since a geometry is regular if and only if it is binary and representable over a field of characteristic not equal to 2 (see [2] or use Tutte's forbidden-minor characterization of regular geometries [16, p. 169]), we can state Heller's theorem as follows: Let $G$ be a binary geometry of rank $n$ which is representable over a field of characteristic not equal to 2. Then the number of points (or rank-1 flats) in $G$ is at most $n(n+1) / 2$.

In this paper we prove an analogue of this theorem for ternary geometries.

(1.1) Theorem. Let $G$ be a ternary geometry of rank $n$ which is representable over a field of characteristic $p$, where $p$ is not equal to 3 . Then the number of points in $G$ is at most $n^{2}$.

We remark that the hypothesis in (1.1) is equivalent to: $G$ is representable over GF(3) and GF( $q)$, where $q$ is a prime power not divisible by 3 . This follows from two results of Rado. The first [14, p. 308] states that a finite geometry is representable over a field $K$ if and only if it is representable over a finite algebraic

* This research was partially supported by National Science Foundation Grant DMS-8521826 and a North Texas State University Faculty Research Grant. 
extension of the prime field of $K$. The second $[14$, p. 309] states that if a finite geometry $G$ is representable over a field of characteristic 0 , then, for all sufficiently large primes $p, G$ is representable over some field of characteristic $p$.

When $p$ is not 2, the upper bound in (1.1) is sharp and is attained by the Dowling geometry $Q_{n}\left(\mathrm{GF}(3)^{\times}\right)$over the multiplicative group $\mathrm{GF}(3)^{\times}$of the field $\mathrm{GF}(3)$ : in fact, except when the rank is 3 , a geometry having the maximum number of points must be a ternary Dowling geometry. We shall prove this in Part II of this series [10]. The Dowling geometry $Q_{n}\left(\mathrm{GF}(3)^{\times}\right)$is the lattice of flats of the cycle geometry of the full signed expansion of the complete graph on $n$ vertices. Thus, it is also the lattice of signed partial partitions on a set of size $n$. See [4] and [20] for details.

The proof of (1.1) is elementary and occupies Sections 2 and 3. Several applications are given in Section 4. Our notation and terminology are for the most part standard (see [17] and [18]) and so we need only note less familiar usages. The characteristic set of a geometry $G$ is the set of characteristics of fields over which $G$ is representable (see p. 160 of [8]). The size $|G|$ of a geometry $G$ is the number of points (or rank-1 flats) in $G$. A line (or rank-2 flat) $\ell$ in $G$ is said to be long if $\ell$ contains at least three points.

Let $G$ be a matroid on the set $S$ and $U \subseteq S$. The deletion $G \backslash U$ is the matroid on the set $S \backslash U$ whose rank function is given by $r_{G \backslash U}(T)=r_{G}(T)$ for $T \subseteq S \backslash U$. The contraction $G / U$ is the matroid on the set $S \backslash U$ whose rank function is given by $r_{G / U}(T)=r_{G}(T \cup U)-r_{G}(U)$ for $T \subseteq S \backslash U$. When $U$ consists of a single element $\omega$, we shall denote the contraction by $G / \omega$. A matroid $M$ is a minor of the matroid $G$ if $M$ can be obtained from $G$ by a sequence of deletions or contractions.

If $G$ is a geometry and $\omega$ is a point in $G$, the circuits of $G / \omega$ are the minimal subsets in the collection $\{C: C$ is a circuit of $G$ not containing $\omega\} \cup\{C \backslash\{\omega\}: C$ is a circuit of $G$ containing $\omega\}$. Thus, if $C$ is a circuit in $G / \omega$, either $C$ or $C \cup\{\omega\}$ is a circuit of $G$. The simplification of $G / \omega$ is the geometry, also denoted by $G / \omega$, on the set of lines incident on $\omega$ whose lattice of flats is the upper interval $[\omega, 1]$. When $G$ is a set of points in projective space, contractions can be represented by projections. More precisely, choose a hyperplane $x$ in projective space not containing $\omega$. The geometry $G / \omega$ can be represented by the projection of the point set $G$ from $\omega$ onto the hyperplane $x$ : that is, $G / \omega$ can be represented by the set of points in $x$ of the form $\ell \wedge x$, where $\ell$ is a line in $G$ incident on $\omega$. The difference $|G|-|G / \omega|$ will be referred to informally as the number of points "destroyed" when $\omega$ is contracted. Because all the points on a long line $\ell$ incident on $\omega$ are identified as one point (namely $\ell$ ) in $G / \omega$, we have

$$
|G|-|G / \omega|=1+\sum_{f}(|\theta|-2)
$$

where the sum is over all long lines $\ell$ in $G$ incident on $\omega$.

We end this section by introducing the key notion in the proof of (1.1). A geometry $G$ is said to be a cone if there exists a point $\omega$ in $G$ such that every other point in $G$ is on a long line incident on $\omega$. The vertex $\omega$ is called an apex of $G$. 
(1.2) Lemma. Let $G$ be a cone with apex $\omega$ and let $a$ be a point in $G$ not equal to $\omega$. Then the contraction $G / a$ (considered as a geometry) is a cone with apex $\omega \vee a$.

Proof. Let $b \vee a$ be a point in the geometry $G / a$ not equal to $\omega \vee a$. Since $G$ is a cone, $b \vee \omega$ is a long line in $G$ and contains a third point $c$. The points $b \vee a$, $\omega \vee a$, and $c \vee a$ are distinct and collinear in $G / a$ and hence, $b \vee \omega \vee a$ is a long line in $G / a$.

\section{A Catalogue of Planar Ternary Geometries}

For easy reference we collect in this section all the information we need about planar (or rank-3) ternary geometries.

Our first goal is to describe all the nonisomorphic planar ternary geometries $G$ with nine points. The best way to do this is to consider the complement of $G$ in the ternary projective plane $\operatorname{PG}(2,3)$. Since $\operatorname{PG}(2,3)$ contains thirteen points, the complement $G^{\mathrm{c}}$ of $G$ contains four points. There are three cases.

(2.1) If $G^{\mathrm{c}}$ is a 4-point line, then $G$ is isomorphic (by definition) to the ternary affine plane $\operatorname{AG}(2,3)$. None of the lines in $\operatorname{AG}(2,3)$ is a 4-point line. Since every point is on four 3-point lines and $\operatorname{AG}(2,3)$ contains exactly nine points, $\operatorname{AG}(2,3)$ is a cone in which every point is an apex. The characteristic set of $\operatorname{AG}(2,3)$ equals the union of $\{0\}$ and the set of primes. An explicit representation over any field in which $\xi^{2}+\xi+1=0$ has a solution can be obtained using the fact that the nine points of inflexion of a nonsingular cubic curve in the complex projective plane form a geometry isomorphic to $\mathrm{AG}(2,3)$ (see p. 163 of $[8]$ ).

(2.2) If $G^{\mathrm{c}}$ is the union of a 3-point line $\ell$ and a point outside $\ell$, then $G$ is isomorphic to the (ternary) Reid geometry $R$, shown in Fig. 1. The Reid geometry $R$ is formed by taking the union of two 4-point lines $\ell_{1}$ and $\ell_{2}$ and one 3-point line $\ell_{3}$ such that their intersection $\ell_{1} \cap \ell_{2} \cap \ell_{3}$ is a point $\omega$. Since the point $\omega$ is on a long line with every other point in $R, R$ is a cone with apex $\omega$. Note that the points $(0,1,1)$ and $(1,1,1)$ in $R$ are also apexes.

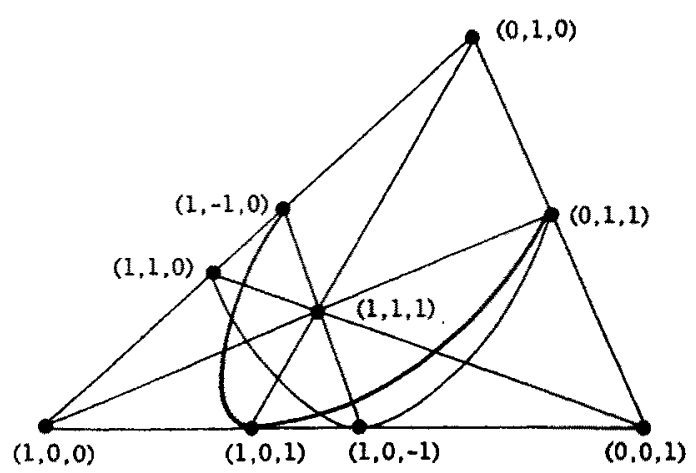

Fig. 1. The Reid geometry. 
(2.2.1) Lemma (Reid). The Reid geometry $R$ has characteristic set $\{3\}$.

Sketch of proof. Assign coordinates. If three points are collinear, then the determinant of the vectors representing them is zero. The long lines in $R$ yield a set of algebraic equations in the coordinates from which we can deduce that 3 equals 0.

An alternate method is to observe that $R$ is isomorphic to the geometry formed by eight of the nine points of inflexions of a nonsingular cubic curve and a point at infinity. We can now use a variation on the argument given in [8,pp. 163-164] to deduce that 3 equals 0 in any field over which $R$ is representable.

Reid's work was unpublished; an account can be found in [6].

(2.3) $G^{\mathrm{c}}$ is a circuit of size 4 in $\operatorname{PG}(2,3)$. Since any two representations of a given ternary geometry over $\mathrm{GF}(3)$ are projectively equivalent (see [3] and [15]), we may take $G^{\mathrm{c}}$ to be the set $\{(1,1,1),(-1,1,1),(1,-1,1),(1,1,-1)\}$ consisting of all the three-dimensional vectors with all coordinates nonzero relative to the natural basis. Thus, $G$ consists of the vectors with exactly one or exactly two nonzero coordinates, that is, $G$ is the ternary planar Dowling geometry $Q_{3}$, or, to give it its full designation, $Q_{3}\left(G F(3)^{\times}\right)$(see [4]). The Dowling geometry $Q_{3}$ is not a cone. Since $Q_{3}$ is representable over any field whose multiplicative group contains the cyclic group of order 2 as a subgroup $[4$, p. 80$]$, its characteristic set is the union of $\{0\}$ and the set of odd primes.

The next goal is to describe all the nonisomorphic planar ternary geometries with 10 points. There are two such geometries.

(2.4) If $G^{\mathrm{c}}$ is a 3-point line, then $G$ is isomorphic to the geometry $R^{+}$obtained by adding a fourth point to the line $\ell_{3}$ in the Reid geometry $R$. Note that $R^{+}$ does not contain $Q_{3}$ as a subgeometry.

(2.5) If $G^{\mathrm{c}}$ consists of three independent points, then $G$ is isomorphic to the geometry $Q_{3}^{+}$obtained by adding one point, say, $(1,1,1)$, to the Dowling geometry $Q_{3}$. Note that $Q_{3}^{+}$contains $R$ as a subgeometry.

Summarizing the results in this section we have

\section{(2.6) Proposition}

(a) Let $G$ be a planar ternary geometry with at least nine points. If $G$ is a cone, then $G$ contains $\operatorname{AG}(2,3)$ or $R$. If $G$ is a cone and contains a 4-point line as a subgeometry, then $G$ contains $R$.

(b) Let $G$ be a planar ternary geometry with at least ten points. Then $G$ contains $R$. 


\section{Cones}

Our proof of (1.1) rests on the following theorem.

(3.1) Theorem. Let $M$ be a ternary geometry satisfying the following property:

$$
M \text { contains a point } \omega \text { such that }|M|-|M / \omega| \geq 2 \operatorname{rank}(M) \text {. }
$$

Then $M$ contains the Reid geometry $R$ as a minor.

Proof. Let $G$ be a minor of $M$ with minimum rank and size satisfying (*) with the point $\omega$. Since $G$ has minimum size, every point in $G$ is contained in a long line incident on $\omega$ (otherwise, we can obtain a smaller minor by deleting any point incident on a 2 -point line with $\omega$ ). Thus, $G$ is a cone with apex $\omega$.

(3.1.1) Lemma. The matroid $G / \omega$ obtained by contracting $\omega$ in $G$ is connected.

Proof. Suppose not. Let $G / \omega=N_{1} \oplus N_{2}$ be a nontrivial separation of the matroid $G / \omega$. Let $n$ be the rank of $G$ and let $n_{i}$ be the rank of $N_{i}$ in $G / \omega$. Observe that

$$
r\left(N_{i} \cup\{\omega\}\right)=n_{i}+1
$$

and

$$
n_{1}+n_{2}=\operatorname{rank}(G / \omega)=n-1
$$

Let $m_{i}$ be the number of points destroyed in $N_{i} \cup\{\omega\}$ when $\omega$ is contracted. We claim that for at least one $i, i=1$ say, $m_{i} \geq 2 n_{i}+2$. (To see this, observe that because $N_{1}$ and $N_{2}$ are disjoint and a total of at least $2 n$ points are destroyed when $\omega$ is contracted,

$$
\left(m_{1}-1\right)+\left(m_{2}-1\right)+1 \geq 2 n .
$$

If $m_{i} \leq 2 n_{i}+1$ for $i=1$ and $i=2$, then

$$
2 n+1 \leq m_{1}+m_{2} \leq 2\left(n_{1}+n_{2}\right)+2=2(n-1)+2=2 n,
$$

a contradiction.) Thus, at least $2 \operatorname{rank}\left(G \backslash N_{2}\right)$ points are destroyed in the deletion $G \backslash N_{2}$ when $\omega$ is contracted, contradicting the assumption that $G$ has minimum size.

(3.1.2) Lemma. Suppose that there are at least two 4-point lines incident on $\omega$. Then $G$ contains $R$ as a minor. 
Proof. Let $\ell_{1}$ and $\ell_{2}$ be two 4-point lines incident on $\omega$. For each line $\ell_{i}$, let $p_{i}$ be a point on $\ell_{i}$ not equal to $\omega$. Since $G / \omega$ is connected, there exists a circuit in $G / \omega$ containing $p_{1}$ and $p_{2}$ (see, for example, p. 108 of [18]). We conclude that either (a) there exists a circuit $\left\{\omega, p_{1}, p_{2}, a_{3}, a_{4}, \ldots, a_{k}\right\}$ in $G$ containing $p_{1}, p_{2}$, and $\omega$, or (b) there exists a circuit $\left\{p_{1}, p_{2}, a_{3}, a_{4}, \ldots, a_{k}\right\}$ containing $p_{1}$ and $p_{2}$, but not $\omega$.

Suppose (a) holds. In the contraction $G /\left\{a_{4}, \ldots, a_{k}\right\}$, the points $\omega, p_{1}, p_{2}, a_{3}$ remain distinct and form a set of rank 3 . Hence, $a_{3}$ lies on the plane $\ell_{1} \vee \ell_{2}$ spanned by the lines $\ell_{1}$ and $\ell_{2}$. As $\omega \vee a_{3}$ is a long line in $G$, it remains a long line in $G /\left\{a_{4}, \ldots, a_{k}\right\}$. Thus, the plane $\ell_{1} \vee \ell_{2}$ contains a third long line, and hence, contains $R$ as a subgeometry.

Suppose (b) holds. If the point $a_{3}$ remains distinct from $\omega$ in $G /\left\{a_{4}, \ldots, a_{k}\right\}$, then, as in (a), the plane $\ell_{1} \vee \ell_{2}$ contains $R$ as a subgeometry. If $\omega$ and $a_{3}$ are identified, consider the sequence of contractions: $G /\left\{a_{k}\right\}$, $G /\left\{a_{k}, a_{k-1}\right\}, \ldots, G /\left\{a_{4}, \ldots, a_{k}\right\}$. Then for some $i \geq 4,\left\{\omega, a_{3}, a_{i}\right\}$ is a circuit in $G /\left\{a_{i+1}, \ldots, a_{k}\right\}$. Hence, there exists a circuit $C$ containing $\omega$ contained in $\left\{\omega, a_{3}, a_{i}, \ldots, a_{k}\right\}$. Eliminating $a_{3}$ from the circuits $C$ and $\left\{p_{1}, p_{2}, a_{3}, a_{4}, \ldots, a_{k}\right\}$, we obtain a circuit $D$ containing $\omega$. In addition to $\omega$, the new circuit $D$ contains both $p_{1}$ and $p_{2}$ (otherwise, we can eliminate $\omega$ from $D$ and $\left\{\omega, a_{3}, a_{i}, \ldots, a_{k}\right\}$ to obtain a circuit which is a proper subset of $\left.\left\{p_{1}, p_{2}, a_{3}, \ldots, a_{k}\right\}\right)$. Thus, (a) holds, and hence, $G$ contains $R$ as a minor.

By (3.1.2) we may now assume that there exists at most one 4-point line incident on $\omega$. Suppose first that there is exactly one 4-point line incident on $\omega$. Because exactly one point other than $\omega$ is destroyed on each 3-point line incident on $\omega$ when $\omega$ is contracted and the number of points destroyed is at least $2 n$, the number of 3-point lines incident on $\omega$ is at least $2 n-3$. Thus, $G$ contains at least $4 n-2$ points. Similarly, if no 4 -point line is incident on $\omega, G$ contains at least $4 n-1$ points. In either case, $G$ contains at least $4 n-2$ points. Thus, to finish the proof of $(3.1)$, it suffices to prove the following lemma.

(3.2) Lemma. Let $G$ be a ternary geometry satisfying:

$$
G \text { is a cone and }|G| \geq 4 \operatorname{rank}(G)-2 \text {. }
$$

\section{Then $G$ contains $R$ as a minor.}

Proof. Let $H$ be a minor of $G$ with minimum rank satisfying ( $\dagger$ ) and let $\omega$ be an apex of $H$. Since a ternary geometry of rank 2 contains at most four points, $\operatorname{rank}(H) \geq 3$. Because $H$ has minimum rank, the contraction $H / p$ does not satisfy $(\dagger)$ for any point $p$ in $H$. From this and (1.2), we conclude that

\section{(3.2.1) Contraction by any point $p$ in $H \backslash \omega$ destroys at least five points.}

Let $a$ be a point in $H \backslash \omega$. By (3.2.1) there exists a long line $\ell$ incident on $a$ not containing $\omega$. Let $a, b, c$ be three distinct points on $\ell$. (There may be a fourth point on $\ell$.) Consider the plane $\Pi$ spanned by $\omega, a$, and $c$. As $\omega$ is an apex, there exist three other points $a^{\prime}, b^{\prime}, c^{\prime}$ on $\Pi$. See Fig. 2. 


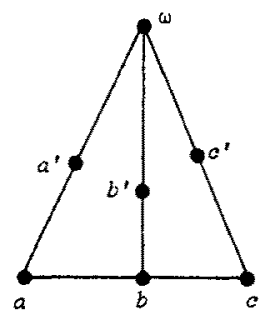

Fig. 2

(3.2.2) If there exists a 4-point line $\ell$ in the plane $\Pi$ not containing $\omega$, then $I I$ contains $R$ as a subgeometry.

Proof. Let $e_{1}, e_{2}, e_{3}, e_{4}$ be the points on the 4-point line $\ell$. As $\omega$ is an apex, there exist at least three points on each of the lines $\omega \vee e_{i}$. Thus, $|\Pi| \geq 9$. As $\Pi$ is a cone and contains a 4-point line, $\Pi$ contains $R$ by (2.6).

A point $p$ in $\Pi$ is said to be inner if all the points destroyed in $H$ when $p$ is contracted lie on $\Pi ; p$ is said to be outer otherwise.

(3.2.3) Let $p$ be an inner point on $\Pi$. Then, either $H$ contains $R$ as a minor, or, $\omega \vee p$ is a 4-point line.

Proof. We distinguish two cases.

Case 1. $\Pi / p$ is a 3-point line. By (3.2.1) there are at least seven points distinct from $p$ in $\Pi$. However, $p$ is incident on three lines. Thus, one of these lines $\ell$ is a 4-point line. If $\ell$ is incident on $\omega$, then $\omega \vee p$ is a 4-point line. If $\ell$ is not incident on $\omega$, then $\Pi$ contains $R$ by (3.2.2).

Case 2. $\Pi / p$ is a 4-point line. By (3.2.1) $|\Pi|-|\Pi / p| \geq 5$ and hence $|\Pi| \geq 9$. Since $\Pi$ is a cone with apex $\omega, \Pi$ contains $\operatorname{AG}(2,3)$ or $R$ by $(2.6)$. We need only consider the case when $\Pi$ is isomorphic to $\operatorname{AG}(2,3)$.

(3.2.4) The set $\Pi \backslash \omega$ does not form a connected component of the matroid $H / \omega$.

Proof. Suppose that $\Pi \backslash \omega$ is a connected component of $H / \omega$. Let $N$ be the set-theoretic complement of $\Pi \backslash \omega$ in $H$ and $r$ be the rank function in $H$. Note that as $|H| \geq 9, N$ is nonempty and $r(N) \geq 1$. Then,

$$
[r(N)-1]+[r(\Pi)-1]=\operatorname{rank}(H / \omega)=\operatorname{rank}(H)-1
$$

Hence, $r(N)=\operatorname{rank}(H)-2$. Since $|N|=|H|-8$,

$$
|N| \geq[4 \operatorname{rank}(H)-2]-8=4 r(N)-2 \text {. }
$$

Since $\omega$ is an apex of $N$, the subgeometry $N$ satisfies $(\dagger)$, contradicting the assumption that $H$ has minimum rank. 
Let $s$ be a point not in $\Pi$ but in the same connected component of $\Pi \backslash \omega$ in $H / \omega$. There exists a circuit in $H / \omega$ containing $s$ and a point in $\Pi \backslash \omega$. Among such circuits, choose one, $C$, of minimum size. Let $d_{1}, d_{2}, \ldots, d_{k}$ be the points in $C$ not in the plane $\Pi$ and consider the sequence of contractions

$$
H /\left\{d_{1}\right\}, H /\left\{d_{1}, d_{2}\right\}, \ldots, H /\left\{d_{1}, d_{2}, \ldots, d_{k}\right\}
$$

Suppose that the point $s$ remains distinct from any of the points in $\Pi$ in $H /\left\{d_{1}, d_{2}, \ldots, d_{k}\right\}$. Then, $\left(C \backslash\left\{d_{1}, d_{2}, \ldots, d_{k}\right\}\right) \cup\{\omega\}$ is dependent and $s$ is in the closure of $\Pi$ in $H /\left\{d_{1}, d_{2}, \ldots, d_{k}\right\}$. Thus, $\Pi$ contains at least ten points and contains $R$ by (2.6).

Now suppose that $s$ is identified with a point $t$ in $\Pi$ in $H:\left\{d_{1}, d_{2}, \ldots, d_{k}\right\}$. Then, for some $i \leq k$ in the sequence of contractions, $\left\{s, t, d_{i}\right\}$ is a circuit in $H /\left\{d_{1}, \ldots, d_{i-1}\right\}$. From this we conclude that $s \vee t$ is a long line not containing $\omega$; otherwise, $\left\{\omega, s, t, d_{1}, \ldots, d_{i-1}\right\}$ is dependent, contradicting the choice of $C$. Consider the plane $\Sigma$ spanned by $\omega, s$, and $t$. As $\omega$ is an apex in $H /\left\{d_{1}, \ldots, d_{i-1}\right\}$, there exists a point $s^{\prime}$ on the line $\omega \vee s$ and a point $d_{i}^{\prime}$ on the line $\omega \vee d_{i}$.

(3.2.5) At least one of the contractions $\Sigma / s$ or $\Sigma / s^{\prime}$ is a 4-point line.

Proof. Let $t^{\prime}$ be a third point on the long line $\omega \vee t$. Suppose $\Sigma / s$ and $\Sigma / s^{\prime}$ are 3-point lines, then the sets $\left\{s, d_{i}^{\prime}, t^{\prime}\right\},\left\{s^{\prime}, d_{i}, t^{\prime}\right\}$, and $\left\{s^{\prime}, d_{i}^{\prime}, t\right\}$ are collinear and $\Sigma$ contains the Fano plane as a subgeometry. However, the Fano plane has characteristic set $\{2\}$ (see p. 533 of [19]), a contradiction.

By (3.2.5) and the fact that all the lines in $\mathrm{AG}(2,3)$ contain exactly three points, contraction by $s$ or $s^{\prime}$ projects a tenth point onto the plane $\Pi$ in $H /\left\{d_{1}, \ldots, d_{i-1}\right\}$. Hence, by (2.6), $H$ contains $R$ as a minor. This completes the proof of (3.2.3).

Using (3.2.3) we can dispose of the case: Two of the points $a, b, c$ in $\Pi$, $a$ and $c$, say, are inner. Applying (3.2.3) to $a$ and $c$, we conclude that either $\Pi$ contains $R$, or, both $\omega \vee a$ and $\omega \vee c$ are 4-point lines. But if the latter holds, $\Pi$ contains $R$.

We can now assume: Two of the points in $\Pi$, a and $c$, say, are outer. Let $\ell_{a}$ and $\ell_{c}$ be long lines incident on $a$ and $c$ not contained in the plane $\Pi$. Let $a, e$, $f$ be three points on $\ell_{a}$ and let $\Sigma=\omega \vee a \vee f$. As $\omega$ is an apex in $H$, we conclude that there are points $e^{\prime}$ and $f^{\prime}$ distinct from $\omega$, $e$, or $f$, on the long lines $\omega \vee e$ and $\omega \vee f$.

(3.2.6) If $\Pi, \ell_{a}$, and $\ell_{c}$ are in general position, that is, $\Pi \vee \ell_{a} \vee \ell_{c}$ has rank 5 , then $H$ contains $R$ as minor.

Proof. Applying (3.2.5) to the points $f$ and $f^{\prime}$ in $\Sigma$, we conclude that $\Sigma / f$, say, is a 4-point line. Hence, in $H / f$, the line $\omega \vee a$ contains a fourth point projected by $f$ from $\Sigma$ onto $\Pi$. Since $\Pi \vee \ell_{a} \vee \ell_{c}$ has rank $5, \ell_{c}$ is still a line outside $\Pi$ in $H / f$. Applying the preceding argument to $\ell_{c}$ in $H / f$, we obtain a fourth point on $\ell_{c}$. We conclude that $H$ contains $R$ as a minor. 
By (3.2.6) we can now assume: $\Pi \vee \ell_{a} \vee \ell_{c}$ has rank 4.

We first consider the case: The point $b$ is inner. By (3.2.3) we can assume that $\omega \vee b$ is a 4-point line. By (3.2.6), $\omega \vee a$ is a 4-point line in, say, $H / f$. Thus, the plane $\Pi$ in the contraction $H / f$ contains $R$ as a subgeometry.

Using the same argument we conclude that if $a^{\prime}, b^{\prime}$, or $c^{\prime}$ is inner, then $H$ contains $R$ as a minor. Thus, we can assume: All the points $a, b, c, a^{\prime}, b^{\prime}, c^{\prime}$ are outer. Let $r$ be one of the points $b, c, a^{\prime}, b^{\prime}$, or $c^{\prime}$. Let $\ell_{r}$ be a long line incident on $r$ not contained in the plane $\Pi$. By (3.2.6) we can assume that $\Pi \vee \ell_{\alpha} \vee \ell_{c}$ contains $\ell_{r}$. Represent the points in $\Pi \vee \ell_{a} \vee \ell_{c}$ in three-dimensional ternary projective space. The line $\ell_{r}$ intersects the plane $\Sigma$ at a point $p$ in $\operatorname{PG}(3,3)$ which may or may not be in $H$.

Suppose first that the point $p$ is not in $H$. As $\Sigma$ contains at least seven points and $p$ is incident on at most four lines in $\Sigma$, there exist two points $e$ and $e^{\prime}$ in $H$ such that $p, e, e^{\prime}$ are collinear and the line $p \vee e$ does not contain $\omega$. Further, at least one of the points, $e^{\prime}$, say, is not on the line $\omega \vee a$. Contracting $e^{\prime}$ we obtain a 4-point line $r \vee e$ not incident on $\omega$ on the plane $\Pi$ in $H / e^{\prime}$. By (3.2.2) $H$ contains $R$ as a minor.

We have now arrived at the last case: All the lines $\ell_{r}$, where $r=b, a^{\prime}, b^{\prime}, c^{\prime}$, intersect the plane $\Sigma$ at a point in $H$. By (3.2.5) applied to $a$ and $a^{\prime}$ in $\Pi$, one of the contractions $\Pi / a$ or $\Pi / a^{\prime}$ is a 4-point line. If $\Pi / a^{\prime}$ is a 4-point line but $\Pi / a$ is not, relabel the points $a, b, c, a^{\prime}, b^{\prime}, c^{\prime}$ in the following way. Since $\Pi$ contains at least seven points, $a^{\prime}$ is on a 3-point line $\ell$ not containing $\omega$. Relabel $a^{\prime}$ by $a$ and relabel the two points on $\ell$ by $b$ and $c$. Now label the remaining points by $a^{\prime}, b^{\prime}, c^{\prime}$ in accordance with Fig. 2.

\section{(3.2.7) Either $|\Sigma / a|=3$ or $H$ contains $R$ as a minor.}

Proof. Suppose $|\Sigma / a|=4$. Since $|\Pi / a|=4$, the plane $\omega \vee \ell_{c}$ in $H / a$ contains two 4-point lines incident on $\omega$, namely, the projections of $I I$ and $\Sigma$, as well as a long line $\ell_{c}$ not incident on $\omega$. As $\omega$ is an apex in $H / a$ by (1.2), $\omega \vee \ell_{c}$ contains $R$ as a subgeometry.

By (3.2.7) we can assume that every point on $\Sigma$ is contained in a 3-point line incident on $a$. Relabeling the points in $\Sigma$ if necessary, let $f$ be the intersection of the line $\ell_{c}$ with the plane $\Sigma$ and let $f^{\prime}$ be another point on the long line $\omega \vee f$. By (3.2.5) at least one of $\Sigma / f$ or $\Sigma / f^{\prime}$ is a 4-point line.

Let $\Sigma^{\prime}$ be the plane $\omega \vee \ell_{c}$. Applying (3.2.5) to the points $f$ and $f^{\prime}$ on the plane $\Sigma^{\prime}$ we conclude that at least one of $\Sigma^{\prime} / f$ or $\Sigma^{\prime} / f^{\prime}$ is a 4-point line. If both $\Sigma / f$ and $\Sigma^{\prime} / f$, or, both $\Sigma / f^{\prime}$ and $\Sigma^{\prime} / f^{\prime}$ are 4-point lines, then, by the argument in (3.2.7), $H$ contains $R$ as a minor. Thus, we may assume (after relabeling) that $|\Sigma / f|=3,\left|\Sigma^{\prime} / f\right|=4,\left|\Sigma / f^{\prime}\right|=4$, and $\left|\Sigma^{\prime} / f^{\prime}\right|=3$.

Consider the plane $\ell_{a} \vee \ell_{c}$. It contains, besides $a, c$, and $f$, a point $e$ on the line $\ell_{\alpha}$, a point $g$ on the line $\ell_{c}$, and the point $b$.

(3.2.8) Either the points $e, b, g$ are collinear, or $H$ contains $R$ as $a$ minor.

Proof. If $e, b, g$ are not collinear, then contraction by $e$ projects a fourth point $g$ onto the line $a \vee c$. By (3.2.2) $H / e$ contains $R$ as a subgeometry. 


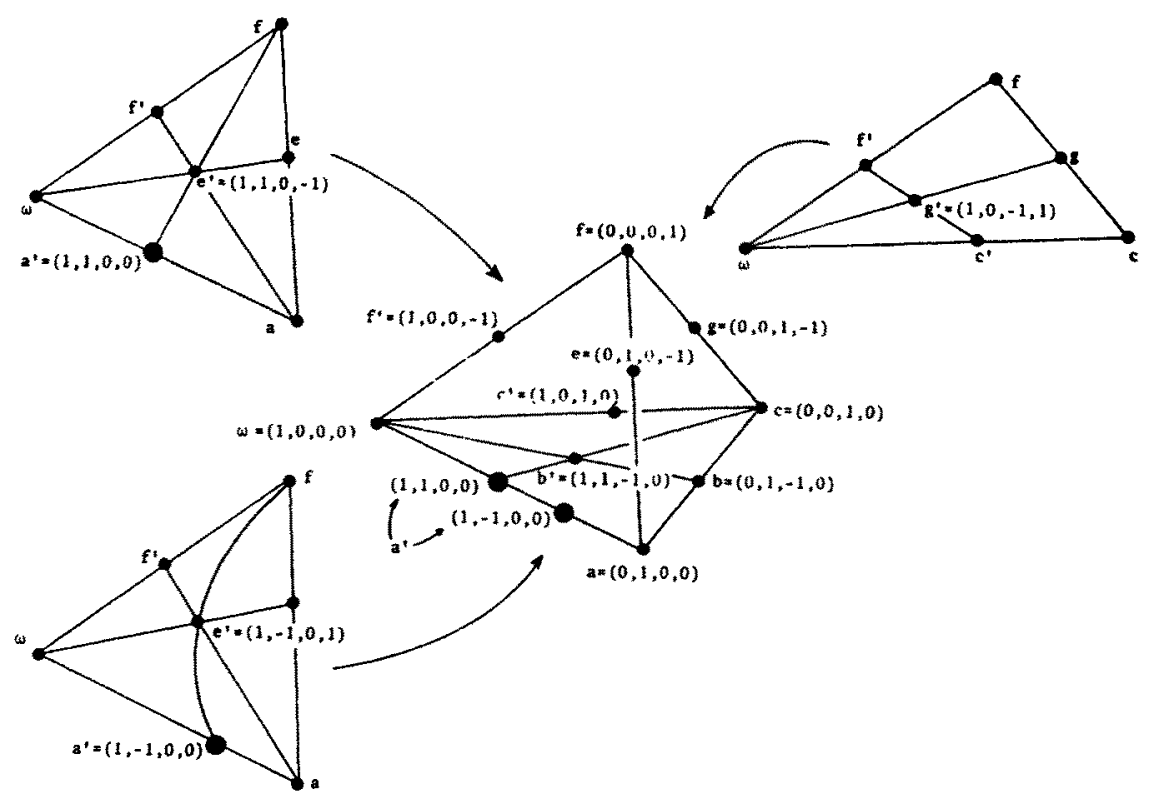

Fig. 3. Assigning coordinates.

By (3.2.8) we can assume that $e, b, g$ are collinear: thus, the plane $\ell_{a} \vee \ell_{c}$ is isomorphic to the cycle geometry $M\left(K_{4}\right)$ of the complete graph on four vertices. Note also that because $\omega$ is an apex, there is a third point $e^{\prime}$ on $\omega v e$ and a third point $g^{\prime}$ on $\omega \vee g$.

To finish, we assign coordinates in $\mathrm{GF}(3)$ to the points in the rank-4 subgeometry $\omega \vee a \vee c \vee f$ as shown in Fig. 3. The assignment is done in the following way. First assign coordinates to the points in the plane $\Pi$. There are two possible choices for the point $a^{\prime}$. Next, assign coordinates to the plane $a \vee f \vee c$. The remaining coordinates are determined by the coordinates that have already been assigned. From Fig. 3 we see that, depending on our choice for $a^{\prime}$, contraction by $c$ or $c^{\prime}$ projects an extra point onto the line $\omega \vee a$ and the line $\omega \vee f$. Thus, $H / c$ or $H / c^{\prime}$ contains $R$ as a subgeometry.

An alternate way to handle the final case is to use the argument in Case 2 of (3.2.3) to project an extra point onto $\Pi \vee \ell_{a} \vee \ell_{c}$. We have presented the configuration proof in detail because we shall need it to prove (5.1).

This completes the proof of (3.2) and (3.1).

We can now use (3.1) to prove a stronger version of (1.1).

(3.3) Theorem. The number of points in a ternary geometry of rank $n$ not containing the Reid geometry $R$ as a minor is at most $n^{2}$. This bound is sharp and is attained by the ternary Dowling geometries. 
Proof. We induct on the rank $n$ of a ternary geometry $G$ not containing $R$ as a minor. If $n \leq 3$, then the theorem holds by (2.6). If $n \geq 4,|G|-|G / a| \leq 2 n-1$ for any point $a$ in $G$ by (3.1). By induction, $|G / a| \leqslant(n-1)^{2}$. Hence, $|G| \leq n^{2}$. To prove the second assertion, observe that the Dowling geometries do not contain $R$ as a minor.

Since the Reid geometry is not representable over a field of characteristic not equal to $3,(1.1)$ follows immediately from (3.3).

\section{Applications}

The Reid geometry is an excluded minor (which is not necessarily minimal) for several minor-closed classes of geometries. One such class is the class of totally dyadic geometries defined independently by Lee [11] and Zaslavsky [21]. (Lee actually defined a more general notion, that of a " $T$-adic" geometry.) A geometry is said to be totally dyadic if it is representable over the rationals by a matrix all of whose subdeterminants take values in the set $\left\{0, \pm 1, \pm 2, \pm 2^{2}, \pm 2^{3}, \ldots\right\}$. Zaslavsky [21] has shown that the subgeometry obtained from $R$ by deleting the apex $\omega$ is not totally dyadic. Since ternary Dowling geometries are totally dyadic, (3.3) holds for a totally dyadic geometry.

Let $\mathscr{A}(p)$ be the minor-closed class of geometries which are algebraic over a field of characteristic $p$ (see p. 14 of [18] for a definition). Gordon [5] has shown that the ternary Reid geometry $R$ is algebraic over a field if and only if that field has characteristic 3. Since ternary Dowling geometries are algebraic over any field (with no restriction on its characteristic), (3.3) holds for a ternary geometry in the class $\mathscr{A}(p)$, where $p$ is a prime not equal to 3 .

\section{Two Technical Remarks}

We record here a technical lemma which will be used in [10].

(5.1) Lemma. Let $G$ be a ternary geometry satisfying: $G$ is a cone and $|G| \geq$ $4 \operatorname{rank}(G)-3$. Then $G$ contairs $\mathrm{AG}(2,3)$ or $R$ as a minor.

Proof. We use the argument in the proof of (3.2). The only change needed is in (3.2.3), which is the only place where the hypothesis that $|G| \geq 4 \operatorname{rank}(G)-2$ is used. By substituting " $H$ contains $\mathrm{AG}(2,3)$ or $R$ " for " $H$ contains $R$ " in the conclusion of (3.2.3) and shortening the proof accordingly, we obtain a proof for $(5.1)$.

The second result is based on the observation that the hypothesis that the geometry is ternary in (3.3) is not really necessary. It suffices only to assume that the geometry does not contain $U_{2,5}$, the 5-point line, or $F_{7}$, the Fano plane, as minors. 
(5.2) Theorem. The number of points in a geometry of rank $n$ not containing $U_{2,5}$, $F_{7}$, or $R$ as minors is at most $n^{2}$. This bound is sharp and is attained by the ternary Dowling geometries.

To prove (5.2) we need to replace arguments in the proof of (3.3) involving coordinates with synthetic incidence arguments. Although this is not difficult, parts of the proof become somewhat complicated. We shall not present the details.

(5.2) extends a result of Oxley [13, Theorem 3.1], which is in turn an extension of (1.1).

\section{Conclusion}

The idea in the proof of (3.1) is to work inside a cone. In hindsight, we can see that this idea was implicit in Heller's paper [7]. Cones can be used in conjunction with long-line graphs (see [9]) to find bounds on the number of points in other kinds of geometries. For example, the number of points in a geometry of rank $n$ representable over $\mathrm{GF}(p)$ and $\mathrm{GF}(q)$, where $p$ and $q$ are coprime prime powers, is at most $c\left(\begin{array}{c}n+1 \\ 2\end{array}\right)$, where $c$ is a constant depending on $p$ and $q$. A proof of this result will appear in a future paper.

\section{Acknowledgments}

I would like to thank Jeff Kahn for several conversations about characteristic sets many years ago. More recently, James Oxley rekindled my interest in the subject. I would like to thank him specially for this, and also for his comments on a first draft of this paper.

\section{References}

1. K. Baclawski and N. L. White, Higher order independence in matroids, J. London Math. Soc. (2) $19(1979), 193-202$.

2. T. Brylawski, A note on Tutte's unimodular representation theorem, Proc. Amer. Math. Soc. 52 (1975), 499-502.

3. T. H. Brylawski and D. Lucas, Uniquely representable combinatorial geometries, in Colloquio Internazionale sulle Teorie Combinatorie, Rome, 1973, Vol. I, 86-104, Accademia Lincei, Rome, 1976.

4. T. A. Dowling, A class of geometric lattices based on finite groups, J. Combin. Theory Ser. B 14 (1973), 61-86; erratum, ibid. 15 (1973), 211.

5. G. Gordon, Algebraic characteristic sets of matroids, J. Combin. Theory Ser, B, in press.

6. C. Greene, Lectures in Combinatorial Geometries, Notes taken by D. Kennedy from the National Science Foundation Seminar in Combinatorial Theory, Bowdoin College, 1971, unpublished.

7. I. Heller, On linear systems with integral valued solutions, Pacific J. Math. 7 (1957), 1351-1354.

8. A. W. Ingleton, Representation of matroids, in Combinatorial Mathematics and Its Applications (D. J. A. Welsh, ed.), 149-169, Academic Press, London and New York, 1971. 
9. J. P. S. Kung, The long-line graph of a combinatorial geometry, I. Excluding $M\left(K_{4}\right)$ and the $(q+2)$-point line as minors, Quart. J. Math. Oxford, in press.

10. J. P. S. Kung and J. G. Oxley, Combinatorial geometries representable over GF(3) and GF( $q$ ). 11. Dowling geometries, Graphs and Combin, in press.

11. J. Lee, Subspaces with well-scaled frames, Ph.D. thesis, Comell University, Ithaca, NY, 1986.

12. U. S. R. Murty, Extremal matroids with forbidden restrictions and minors (synopsis), in Proc. Seventh Southeastern Conf. on Combinatorics, Graph Theory, and Computing, Louisiana State University, Baton Rouge, LA, 1976, 463-468, Congressus Numerantium, No. 17, Utilitas Math., Winnipeg, Manitoba, 1976.

13. J. G. Oxley, On matroids with no 5-point-line-minor, preprint.

14. R. Rado, Note on independence functions, Proc. London Math. Soc. (3) 7 (1957), 300-320.

15. P. D. Seymour, Matroid representation over GF(3). J. Combin. Theory Ser. B $26(1979), 159-173$.

16. W. T Tutte, A homotopy theorem for matroids, II, Trans. Amer. Math. Soc. 88 (1958), 161-175.

17. D. J. A. Welsh, Matroid Theory, Academic Press, London and New York, 1976.

18. N. L. White (ed.), Theory of Matroids, Cambridge University Press, Cambridge, 1986.

19. H. Whitney, On the abstract properties of linear dependence, Amer, J. Math. 57 (1935), 509-533.

20. T. Zaslavsky, Signed graphs, Discrete Appl. Math. 4 (1982), 47-74; erratum, ibid. 5 (1983), 248.

21. T. Zaslavsky, private communication, November 1986.

Received November 20,1986, and in revised form June 22, 1987. 